\title{
INDEX FOR VOLUME 114
}

Adell, J. A. \& De La Cal, J. Limiting properties of inverse Beta and generalized Bleimann-Butzer-Hahn operators .

Arratia, R., Barbour, A. D. \& Tavaré, S. On random polynomials over finite fields . . $\quad 347$

Aupetit, B. Spectral characterization of the radical in Banach and Jordan-Banach algebras 31

Barbour, A. D., Tavaré, S. \& Arratia, R. On random polynomials over finite fields . $\quad .347$

Bennett, M. Fractional parts of powers of rational numbers _ . . . . . . . . . 191

Bowers, P. L. The upper Perron method for labelled complexes with applications to circle packings . . . . . . . . . . . . . . . . . 32

Bravo, F. \& Girela, D. Some results of the Lindelöf type involving the segmental

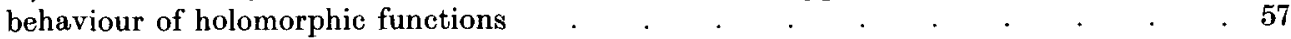

Brown, G., Wang, K.-Y. \& Wilson, D. C. Positivity of some basic cosine sums $\quad$. $\quad .383$

Bryant, R. M. \& Gupta, C. K. Automorphisms of free nilpotent-by-abelian groups . $\quad 143$

Buhmann, M. Discrete least squares approximation and prewavelets from radial function spaces

Bullejos, M., Carrasco, P. \& Cegarra, A. M. Cohomology with coefficients in symmetric cat-groups. An extension of Eilenberg-MacLane's classification theorem . . . 16

Carrasco, P., Cegarra, A. M. \& Bullejos, M. Cohomology with coefficients in symmetric cat-groups. An extension of Eilenberg-MacLane's classification theorem . . . 163

Cassels, J. W. S. Jacobian in genus 2 .

Cegarra, A. M., Bullejos, M. \& Carrasco, P. Cohomology with coefficients in symmetric cat-groups. An extension of Eilenberg-MacLane's classification theorem

De Angelis, D., Hall, P. \& Young, G. A. A note on coverage error of bootstrap confidence intervals for quantiles

De La Cal, J. \& Adell, J. A. Limiting properties of inverse Beta and generalized Bleimann-Butzer-Hahn operators .

Dellnitz, M. \& Melbourne, I. Normal forms for linear Hamiltonian vector fields commuting with the action of a compact Lie group.

Dowling, P. N. \& Lennard, C. J. Uniform Kadec-Klee-Huff properties of vector-valued Hardy spaces.

Fountain, J. \& Lewin, A. Products of idempotent endomorphisms of an independence algebra of infinite rank.

Girela, D. \& Bravo, F. Some results of the Lindelöf type involving the segmental behaviour of holomorphic functions

Gupta, C. K. \& Bryant, R. M. Automorphisms of free nilpotent-by-abelian groups . . 143

Gupta, N. \& Shpilrain, V. Nielsen's commutator test for two-generator groups . $\quad$. $\quad 295$

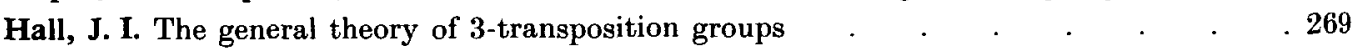

Hall, P., Young, G. A. \& De Angelis, D. A note on coverage error of bootstrap confidence intervals for quantiles . $. \quad . \quad . \quad . \quad . \quad . \quad . \quad . \quad . \quad . \quad . \quad .517$

Hall, R. R. \& Wilson, J. C. On reciprocity formulae for inhomogeneous and homogeneous Dedekind sums

Hansen, J. C. \& Schmutz, E. How random is the characteristic polynomial of a random matrix . . . . . . . . . . . . . . . . 507

Heinonen, J. \& Rohde, S. The Gehring-Hayman inequality for quasihyperbolic geodesics . 393

Higgins, P. J. \& Mackenzie, K. C. H. Duality for base-changing morphisms of vector bundles, modules, Lie algebroids and Poisson structures 
Higson, N., Roe, J. \& Yu, G. A coarse Mayer-Vietoris principle _ . . . . . $\quad$. 85

Hillman, J. A. On 3-dimensional Poincaré duality complexes and 2-knot groups. . $\quad 215$

Johnson, F. E. A. \& Kotschick, D. On the signature and Euler characteristic of certain four-manifolds.

Jordan, D. A. Height one prime ideals of certain iterated skew polynomial rings $\quad . \quad .407$

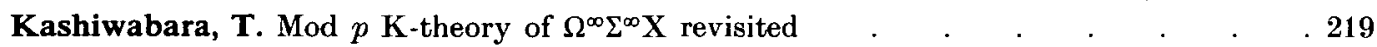

Kondracki, W., Królak, A. \& Rudnicki, W. On the instability of a class of generalized strong curvature singularities. . . . . . . . . . . . . . . . 379

Kotschick, D. \& Johnson, F. E. A. On the signature and Euler characteristic of certain four-manifolds.

Królak, A., Rudnicki, W. \& Kondracki, W. On the instability of a class of generalized strong curvature singularities .

Lennard, C. J. \& Dowling, P. N. Uniform Kadec-Klee-Huff properties of vector-valued Hardy spaces.

Lewin, A. \& Fountain, J. Products of idempotent endomorphisms of an independence

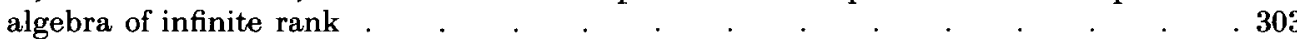

Loos, O. Properly algebraic and spectrum-finite ideals in Jordan systems . . . . 149

Mackenzie, K. C. H. \& Higgins, P. J. Duality for base-changing morphisms of vector bundles, modules, Lie algebroids and Poisson structures _. . . . . . . . 471

Martin, M.-A. \& Mattila, P. Hausdorff measures, Hölder continuous maps and self-similar fractals

Mattila, P. \& Martin, M.-A. Hausdorff measures, Hölder continuous maps and self-similar fractals

Melbourne, I. Versal unfoldings of equivariant linear Hamiltonian vector fields.

Melbourne, I. \& Dellnitz, M. Normal forms for linear Hamiltonian vector fields commuting with the action of a compact Lie group. . . . . . . . . . . . . . 235

Merriman, J. R. \& Smart, N. P. Curves of genus 2 with good reduction away from 2 with a rational Weierstrass point . . . . . . . . . . . . 203

Morris, S. A. \& Pestov, V. G. Open subgroups of free abelian topological groups $\quad . \quad 439$

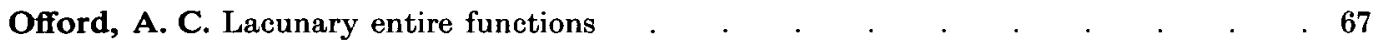

Oguiso, K. A remark on the global indices of Q-Calabi-Yau 3-folds . . . . . . . . 427

Pestov, V. G. \& Morris, S. A. Open subgroups of free abelian topological groups $\quad . \quad 439$

Promislow, S. D. A class of groups producing simple, unique trace $\mathrm{C}^{*}$-algebras . . . 223

Rajeev, B. On asymptotics of a class of 1-dimensional diffusions . $\quad . \quad$. $\quad . \quad$. $\quad . \quad 499$

Robinson, P. L. Equivariant prequantization and admissible coadjoint orbits . . . $\quad .131$

Roe, J., Yu, G. \& Higson, N. A coarse Mayer-Vietoris principle _ . . . . . . $\quad 85$

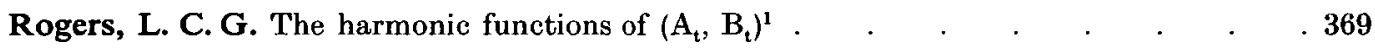

Rognes, J. Characterizing connected K-theory by homotopy groups . . . . . . . . . $\quad .99$

Rohde, S. \& Heinonen, J. The Gehring-Hayman inequality for quasihyperbolic geodesics . 393

Rudnicki, W., Kondracki, W. \& Królak, A. On the instability of a class of generalized

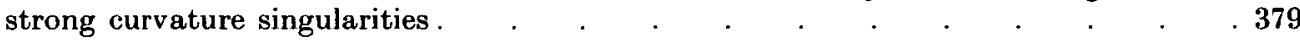

Schmutz, E. \& Hansen, J. C. How random is the characteristic polynomial of a random matrix ? 507

Serrano, F. The sheaf of relative differentials of a fibred surface. _ . _ . . . . . $\quad .461$

Shpilrain, V. \& Gupta, N. Nielsen's commutator test for two-generator groups. . . $\quad 295$

Silver, D. S. On the existence of minimal Seifert manifolds . _ . _ . _ . _ . . . 103

Smart, N. P. \& Merriman, J. R. Curves of genus 2 with good reduction away from 2 with a rational Weierstrass point . . . . . . . . . . . . . . . . . . 203

Stallard, G. M. The iteration of entire functions of small growth _ . . . . . . . . . $\quad$. 43

Sudbery, A. The algebra of differential forms on a full matric bialgebra . . . . . . 111

Tavaré, S., Arratia, R. \& Barbour, A. D. On random polynomials over finite fields . $\quad .347$ 
Turner, P. R. Dyer-Lashof operations in the Hopf ring for complex cobordism . . . . 453

Vitter, A. Hermitian-Einstein metrics and jumping lines for $S p(\mathbf{n}+1)$-homogeneous bundles over $\mathrm{P}^{2 \mathrm{n}+1}$.

Wang, K.-Y., Wilson, D. C. \& Brown, G. Positivity of some basic cosine sums $\quad$. $\quad .383$

Wilson, D. C., Brown, G. \& Wang, K.-Y. Positivity of some basic cosine sums . . 383

Wilson, J. C. \& Hall, R. R. On reciprocity formulae for inhomogeneous and homogeneous Dedekind sums .

Young, G. A., De Angelis, D. \& Hall, P. A note on coverage error of bootstrap confidence intervals for quantiles. . . . . . . . . . . . . . . 517

Yu, G., Higson, N. \& Roe, J. A coarse Mayer-Vietoris principle _ . . . . $\quad$. 85 


\section{The attention of authors is particularly directed to the following requests.}

1. Papers should be typed, double-spaced, on one side of white paper (of which A4, 210 by $297 \mathrm{~mm}$, is a suitable size). The pages must be numbered. Margins of $30 \mathrm{~mm}$ should be left at the side, top and bottom of each page. Two clear copies should be sent.

A cover page should give the title, the author's name and institution, with the address at which mail is to be sent.

The title, while brief, must be informative (e.g. A new proof of the prime-number theorem, whereas Some applications of a theorem of G. H. Hardy would be useless).

The first paragraph or two should form a summary of the main theme of the paper, providing an abstract intelligible to mathematicians.

For a typescript to be accepted for publication, it must accord with the standard requirements of publishers, and be presented in a form in which the author's intentions regarding symbols etc. are clear to a printer (who is not a mathematician).

The following notes are intended to help the author in preparing the typescript. New authors may well enlist the help of senior colleagues, both as to the substance of their work and the details of setting it out correctly and attractively.

\section{Notation}

Notation should be chosen carefully so that mathematical operations are expressed with all possible neatness, to lighten the task of the compositor and to reduce the chance of error.

For instance $n_{k}(n$ sub $k$ ) is common usage, but avoid if possible using $c \operatorname{sub} n$ sub $k$. Fractions are generally best expressed by a solidus. Complicated exponentials like

should be shown in this and no other way.

$$
\exp \left\{z^{2} \sin \theta /\left(1+y^{2}\right)\right\}
$$

In the manuscript, italics, small capitals and capitals are specified by single, double and triple underlinings. Bold faced type is shown by wavy underlining; wavy will be printed wavy.

It helps if displayed equations or statements which will be quoted later are numbered in order on the right of their line. They can then be referred to by, for example, 'from (7)'.

The author must enable the printer (if necessary by pencilled notes in the margin) to distinguish between similar symbols such as $0, O, 0, \mathrm{O}, 0 ; x, \mathrm{X}, \mathrm{x} ; \phi, \Phi, \varnothing ; 1,1 ; \varepsilon, \in ; \kappa, k$.

Greek letters can be denoted by Gk in the margin.

If an author wishes to mark the end of the proof of a theorem, the sign I may be used.

Footnotes should be avoided.

\section{Diagrams}

It is extremely helpful if diagrams are drawn in Indian ink on white card, faintly blue or green-lined graph paper, or tracing cloth or paper. Symbols, legends and captions should be given on a transparent overlay. Each text figure must be numbered as Figure 1, Figure 2, ... and its intended position clearly indicated in the manuscript:

\section{Figure 1 here}

The author's name in pencil must be on all separate sheets of diagrams

A figure is expensive to reproduce and should be included only when the subject matter demands it, or when it greatly clarifies the exposition.

The Society recognizes that some authors do not have the facilities for producing drawings of a sufficiently high standard to be reproduced directly and it is therefore willing to have such diagrams redrawn, provided that they are clear.

\section{Tables}

Tables should be numbered (above the table) and set out on separate sheets. Indicate the position of each in the text as for figures:

Table 3 here

\section{References}

References should be collected at the end of the paper numbered in alphabetical order of the authors' names. Titles of journals should be abbreviated as in Mathematical Reviews. The following examples show the preferred style for references to a paper in a journal, a paper in a proceedings volume, a book and an unpublished dissertation:

[1] J. F. Adams. On the non-existence of elements of Hopf invariant one. Ann. of Math. (2) 72 (1960), 20-104.

[2] M. P. Fourman and D. S. Scott. Sheaves and logic. In Applications of Sheaves, Lecture Notes in Math. vol. 753 (Springer-Verlag, 1979), pp. 302-401.

[3] P. T. Johnstone. Stone Spaces. Cambridge Studies in Advanced Math. no. 3 (Cambridge University Press, 1982).

[4] F. W. Lawvere. Functorial semantics of algebraic theories. Ph.D. thesis. Columbia University (1963). 


\section{Mathematical Proceedings of the Cambridge Philosophical Society \\ MPCPCO II 4 (Pt 3) 383-58I (1993) 0305-004I November 1993}

\section{CONTENTS}

Brown, G., WANG, K.-Y. \& WILson, D. C. Positivity of some basic cosine sums . . . . 383

Heinonen, J. \& RoHde, S. The Gehring-Hayman inequality for quasihyperbolic geodesics. . 393

JORDAN, D. A. Height one prime ideals of certain iterated skew polynomial rings . . . . 407

OGUISo, K. A remark on the global indices of $\mathbb{Q}$-Calabi-Yau 3-folds . . . . . . . . 427

Johnson, F. E. A. \& Kотsеніск, D. On the signature and Euler characteristic of certain fourmanifolds.

Morris, S. A. \& Pestrov, V. G. Open subgroups of free abelian topological groups.

VITTER, A. Hermitian-Einstein metries and jumping lines for $S p(n+1)$-homogeneous bundles over $\mathbb{P}^{2 n+1}$.

.

RER, P. R. Dyer-Lashof operations in the Hopf ring for complex cobordism . . . . $\quad .453$

SERrano, F. The sheaf of relative differentials of a fibred surface . _ . _ . _ . . . . 461

Higgins, P. J. \& Mackenzie, K. C. H. Duality for base-changing morphisms of vector bundles, modules, Lie algebroids and Poisson structures _. . . . . . . . .

Adell, J. A. \& DE LA CAL, J. Limiting properties of Inverse Beta and generalized Bleimann-Butzer-Hahn operators .

RAJEEV, B. On asymptoties of a class of 1-dimensional diffusions . . . . . . . . . .

Hansen, J. C. \& Schmutz, E. How random is the characteristic polynomial of a random matrix?

De Angelis, D., Hall, P. \& Young, G. A. A note on coverage error of bootstrap confidence intervals for quantiles

Buhmann, M. Diserete least squares approximation and prewavelets from radial function spaces .
Melbounne, I. Versal unfoldings of equivariant linear Hamiltonian vector fields .

Prooeedings of Meetings 